\title{
Adipocyte-specific modulation of KLF14 expression in mice leads to sex-dependent impacts on adiposity and lipid metabolism
}

\section{Running title: KLF14 is sex-dimorphic in mouse metabolism}

Qianyi Yang ${ }^{1}$, Jameson Hinkle ${ }^{1}$, Jordan N. Reed ${ }^{1,2}$, Redouane Aherrahrou ${ }^{1}$, Zhiwen Xu ${ }^{3}$, Thurl E. Harris ${ }^{4}$, Erin J. Stephenson ${ }^{5}$, Kiran Musunuru ${ }^{6,7,8}$, Susanna R. Keller ${ }^{9}$, Mete Civelek ${ }^{1,2}$

${ }^{1}$ Center for Public Health Genomics, School of Medicine, University of Virginia, Charlottesville, VA 22908, USA.

${ }^{2}$ Department of Biomedical Engineering, School of Engineering and Applied Science, University of Virginia, Charlottesville, VA 22908, USA.

${ }^{3}$ Department of Chemistry, College of Arts and Sciences, University of Virginia, Charlottesville, VA 22908, USA.

${ }^{4}$ Department of Pharmacology, School of Medicine, University of Virginia, Charlottesville, VA 22908, USA.

${ }^{5}$ Department of Anatomy, College of Graduate Studies \& Chicago College of Osteopathic Medicine, Midwestern University, Downers Grove, IL 60515, USA.

${ }^{6}$ Cardiovascular Institute, Perelman School of Medicine at the University of Pennsylvania, Philadelphia, PA, 19104, USA.

${ }^{7}$ Division of Cardiovascular Medicine, Department of Medicine, Perelman School of Medicine at the University of Pennsylvania, Philadelphia, PA, 19104, USA.

${ }^{8}$ Department of Genetics, Perelman School of Medicine at the University of Pennsylvania, Philadelphia, PA, 19104, USA.

${ }^{9}$ Division of Endocrinology and Metabolism, Department of Medicine, School of Medicine, University of Virginia, Charlottesville, VA, 22908, USA. 


\section{Corresponding authors:}

Qianyi Yang, Ph.D. and Mete Civelek, PhD

University of Virginia

Center for Public Health Genomics

Old Med School 3836

PO Box 800717

Charlottesville, VA 22908-0717

Office Number: 434-243-1669

Fax Number: 434-982-1815

E-mail: qy5sy@virginia.edu and mete@virginia.edu

Word count: 6483

Number of tables: 2 (supplemental)

Number of figures: 8 main figures and 5 supplemental figures 


\section{SUPPLEMENTAL FIGURES:}

\section{Supplemental Figure 1}

A
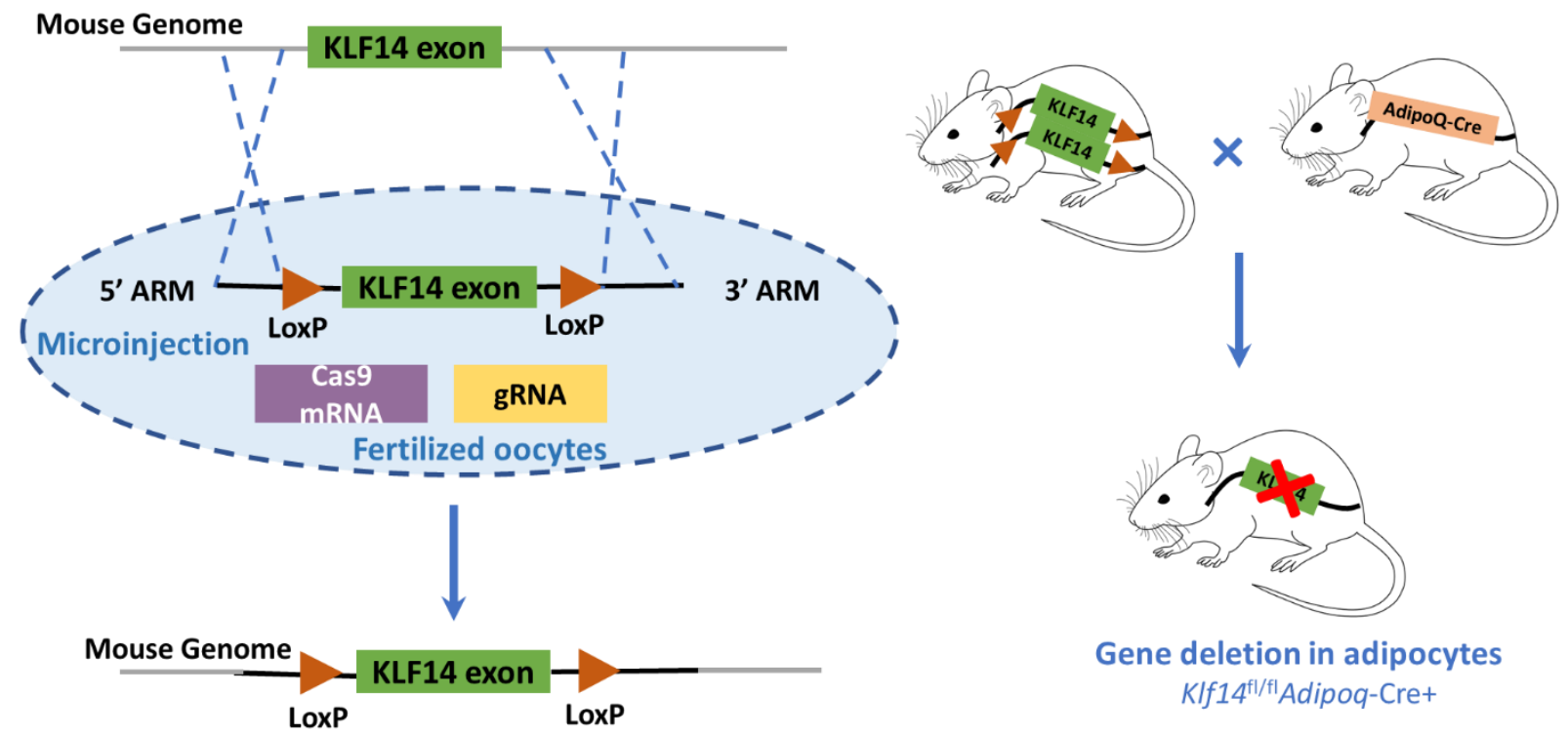

B

IB: KLF14

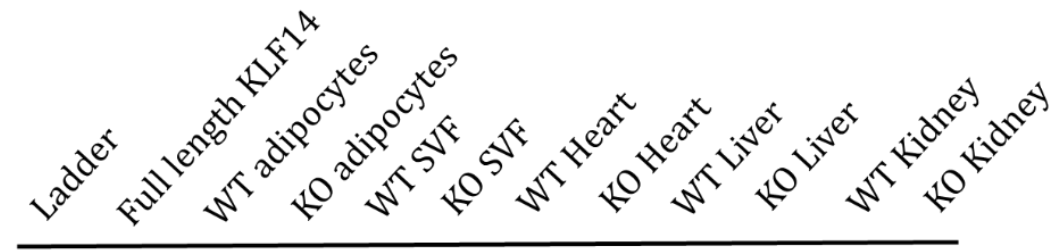

KLF14

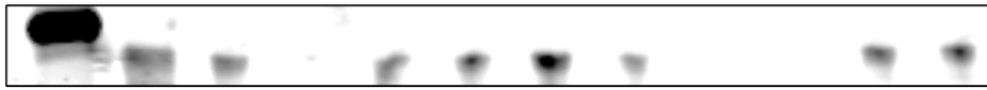

IB: Actin

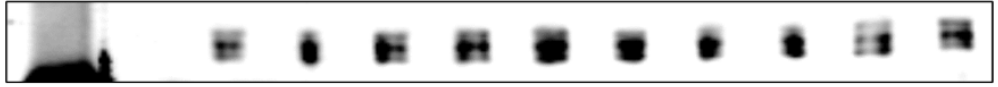

C

IB: KLF14

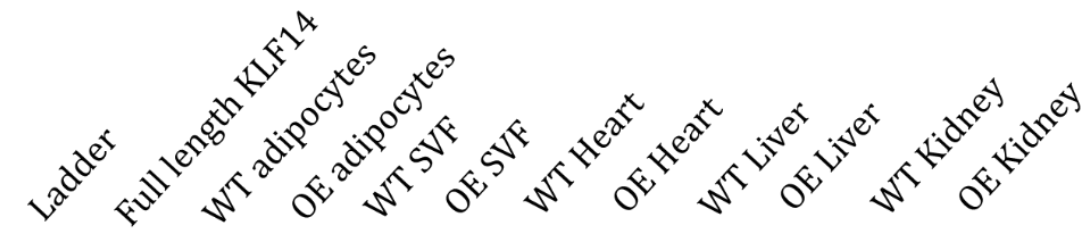

KLF14

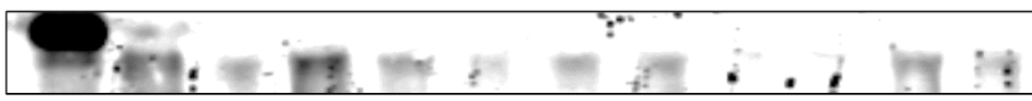

IB: Actin

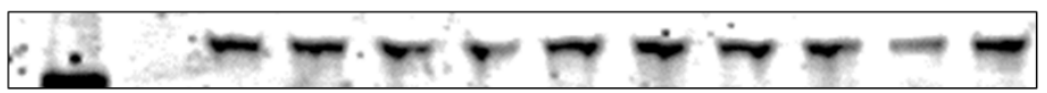


Supplemental Figure 2

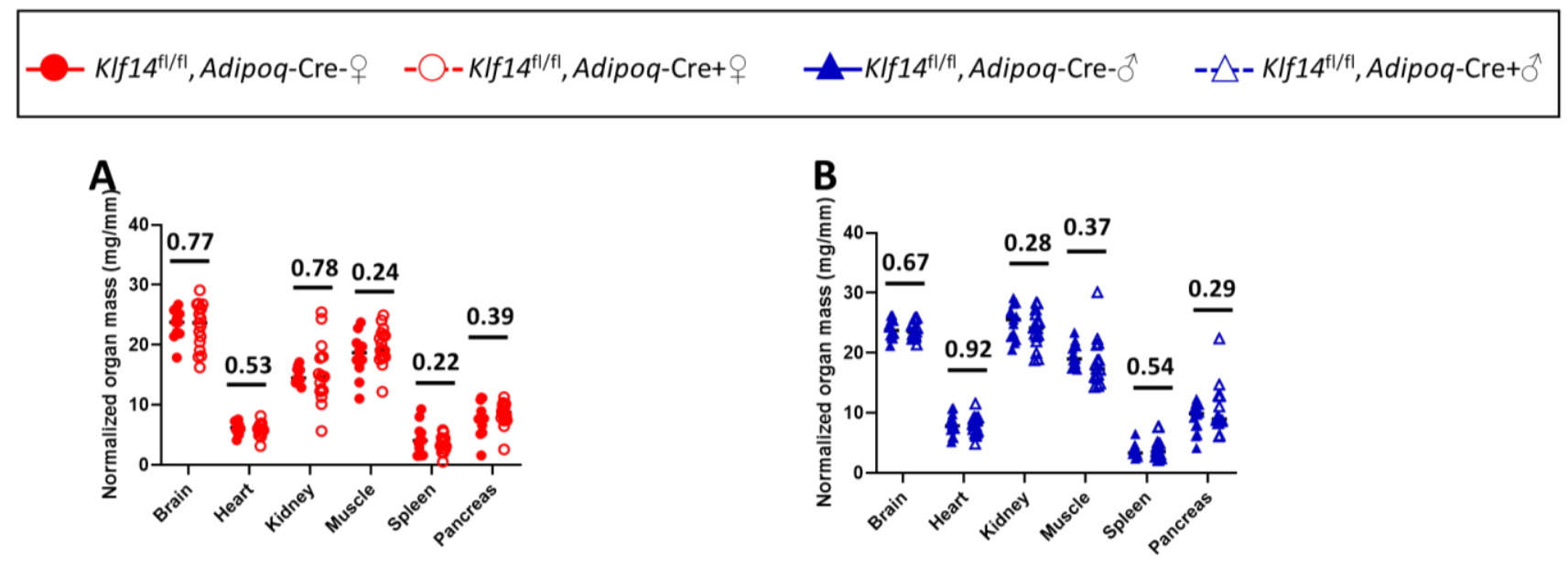


Supplemental Figure 3

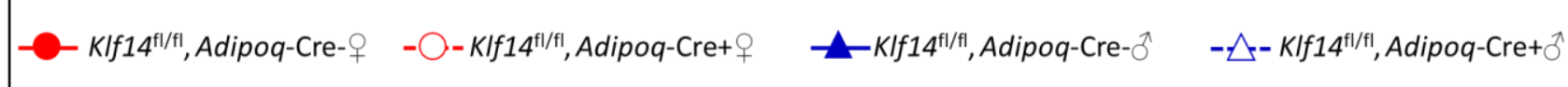

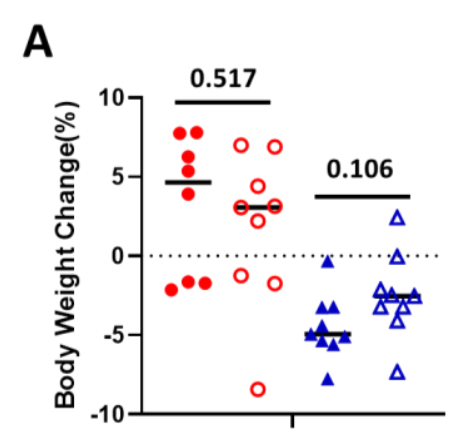

B

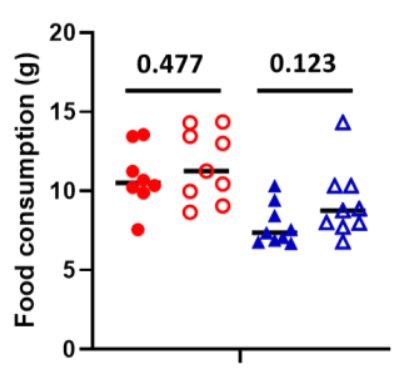




\section{Supplemental Figure 4}

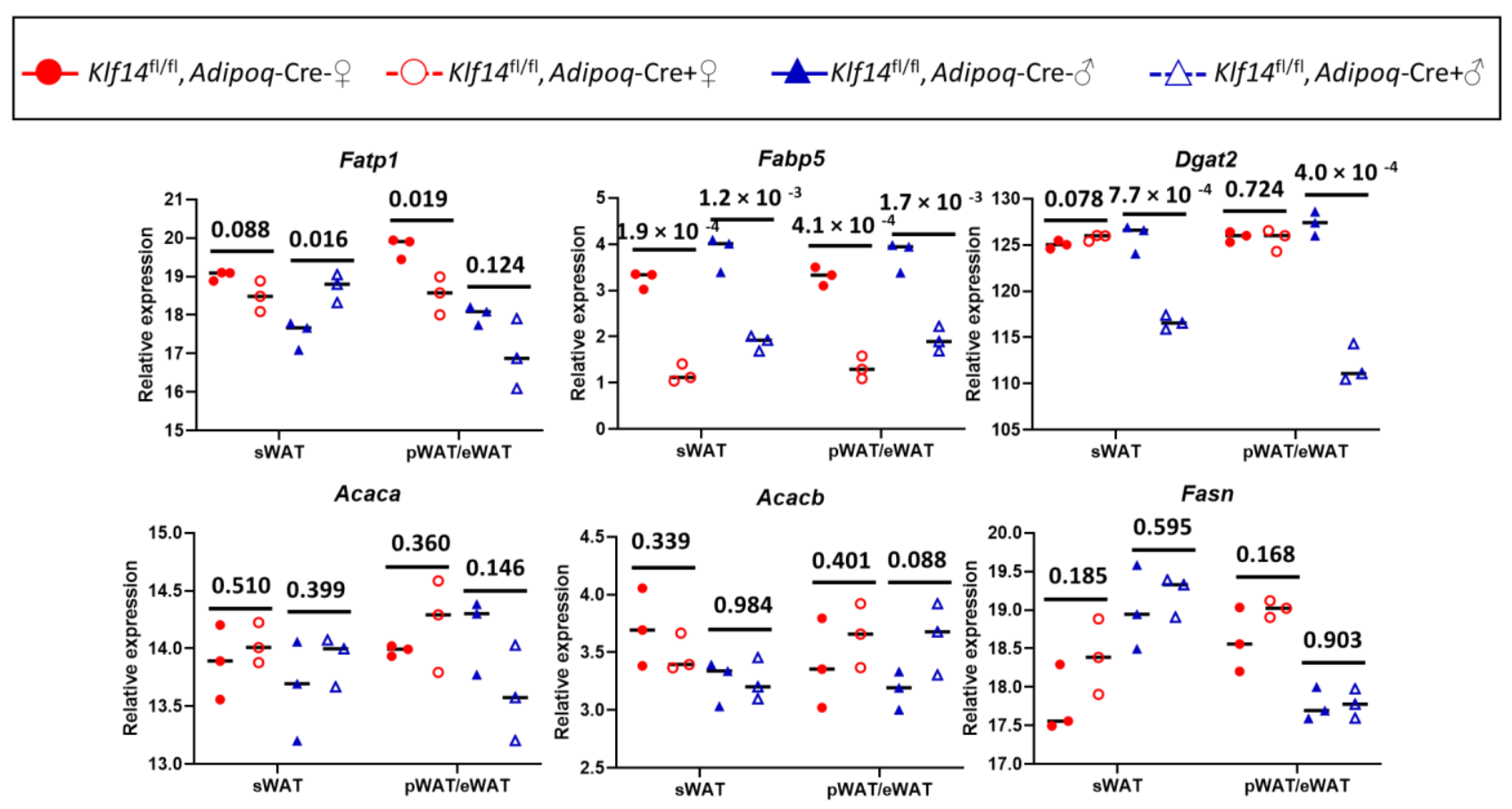




\section{Supplemental Figure 5}

A

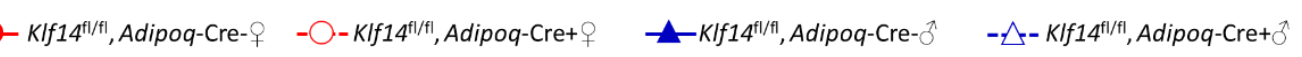
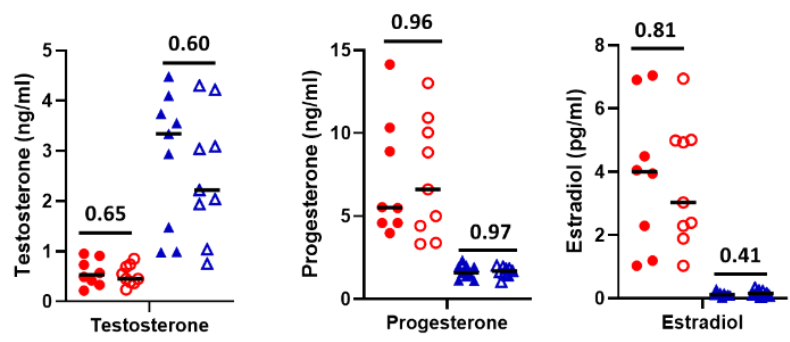

B

C

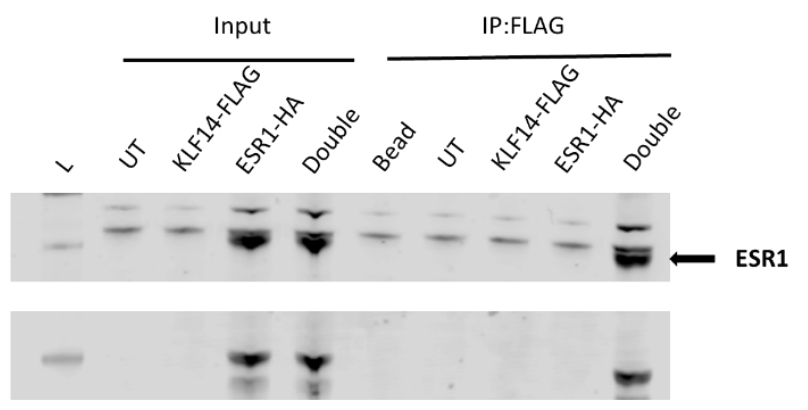

C

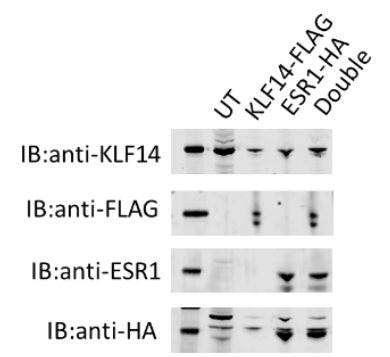

IB:anti-ESR1 


\section{SUPPLEMENTAL FIGURE LEGENDS}

Supplemental Figure 1: Characterization of transgenic mice. (A) Targeting strategy for deletion of Klf14 in mouse adipocytes. (B) KLF14 protein level in adipocytes, stromal vascular fraction (SVF), heart, liver and kidney of wild-type (WT) and knockout (KO) mice. (C) KLF14 protein level in adipocytes, stromal vascular fraction (SVF), heart, liver and kidney of wild-type (WT) and overexpression (OE) mice.

Supplemental Figure 2: Tissue weights normalized to tibia length in adipocyte-specific Klf14 knockout and wild type (A) female and (B) male mice. $\mathrm{N}_{\mathrm{Female} \text {,Adipoq-Cre- }}=11, \mathrm{~N}_{\mathrm{Female}, \text { Adipoq-Cre+ }}=17, \mathrm{~N}_{\mathrm{Male}, \text { Adipoq-Cre- }}=14$, $\mathrm{N}_{\text {Male,Adipoq-Cre+ }}=14$.

Supplemental Figure 3: (A) Body weight change and (B) food consumption during the three-day period in metabolic cages. $\mathrm{N}_{\text {Female_Adipoq-Cre- }}=8, \mathrm{~N}_{\text {Female_Adipoq-Cre+ }}=9, \mathrm{~N}_{\text {Male_Adipoq-Cre- }}=9, \mathrm{~N}_{\text {Male_Adipoq-Cre+ }}=9$.

Supplemental Figure 4: mRNA expression of fatty acid uptake and metabolism genes Fatpl, Fabp5, Dgat2, Acaca, Acacb, Fasn and in isolated mature adipocytes. N =3 mice per genotype and sex. Relative gene expression, normalized to GAPDH levels, was calculated using the $2^{-\Delta \Delta \mathrm{CT}}$ method. $P$-values were calculated using two-tailed unpaired Student's t -test.

Supplemental Figure 5: (A) Sex hormone Testosterone, Progesterone and Estradiol levels in serum of adipocyte Klf14-deficient female and male mice and control littermates at euthanasia after 21 weeks of HFD.

$\mathrm{N}_{\text {Female_Adipoq-Cre- }}=8, \mathrm{~N}_{\text {Female_Adipoq-Cre+ }}=9, \mathrm{~N}_{\text {Male_Adipoq-Cre- }}=9, \mathrm{~N}_{\text {Male_Adipoq-Cre+ }}=9$. (B) KLF14, FLAG, ESR1 and HA levels were measured in HEK293 cells that are untransfected (UT) or transfected with either KLF14-FLAG or ESR1-HA or both. (C)Abovementioned HEK293 cells were immunoprecipitated with FLAG antibodyconjugated magnetic beads. Samples were immunoblotted with anti-ESR1 or anti-HA antibodies. 
Supplemental table 1: Mouse genotyping primer list.

\begin{tabular}{|l|l|l|l|}
\hline Name & Forward & Reverse & Amplicon \\
\hline Adipoq_Klf14_Cre & GAACCTGATGGACATGTTCAGG & AGTGCGTTCGAACGCTAGAGCCTGT & 250bp \\
\hline Adipoq_Klf14_OE & GGCCTACTACAAGTCGTCGC & CCGGGCTGCAGGAATTCGAT & $489 \mathrm{bp}$ \\
\hline
\end{tabular}


Supplemental table 2: qPCR primer list.

\begin{tabular}{|c|c|c|c|c|}
\hline Gene & Forward & Reverse & Accession ID & Reference \\
\hline Fatp1 & GGCTCCTGGAGCAGGAACA & ACGGAAGTCCCAGAAACCAA & NM_011977.4 & $(1)$ \\
\hline Fatp4 & ACGATGTTTCCTGCTGAGTGGTA & CTCTCCGACCTGCCACAGA & NM_011989.5 & $(1)$ \\
\hline Fabp4 & ATGTGCGACCAGTTTGTG & TTTGCCATCCCACTTCTG & NM_024406.3 & $(2)$ \\
\hline Fabp5 & GCTGATGGCAGAAAAACTCAGA & CCTGATGCTGAACCAATGCA & NM_001272098.1 & (3) \\
\hline Acc1 & CCTCCGTCAGCTCAGATACA & TTTACTAGGTGCAAGCCAGACA & NM_133360.2 & (4) \\
\hline Fasn & GCTGCGGAAACTTCAGGAAAT & AGAGACGTGTCACTCCTGGACTT & NM_007988.3 & $(5)$ \\
\hline Acc2 & ACAGAGATTT CACCGTTGCGT & CGCAGCGATGCCATTGT & NM_133904 & $(6)$ \\
\hline Dgat1 & ACCGCGAGTTCTACAGAGATTGGT & ACAGCTGCATTGCCATAGTTCCCT & NM_010046.3 & $(7)$ \\
\hline Dgat2 & TGGGTCCAGAAGAAGTTCCAGAAGTA & ACCTCAGTCTCTGGAAGGCCAAAT & NM_026384.3 & $(7)$ \\
\hline Hsl & GCTGGGCTGTCAAGCACTGT & GTAACTGGGTAGGCTGCCAT & NM_001039507.2 & (8) \\
\hline Atgl & TGTGGCCTCATTCCTCCTAC & TCGTGGATGTTGGTGGAGCT & NM_001163689.1 & $(8)$ \\
\hline Gapdh & СТCССACTCTTCCACCTTCG & GCCTCTCTTGCTCAGTGTCC & NM_001289726.1 & $(9)$ \\
\hline
\end{tabular}


1. Mishima T, Miner JH, Morizane M, Stahl A, Sadovsky Y. The Expression and Function of Fatty Acid Transport Protein-2 and -4 in the Murine Placenta. PLoS One [Internet]. 2011 Oct 20 [cited 2021 Jun 14];6(10). Available from: https://www.ncbi.nlm.nih.gov/pmc/articles/PMC3197585/

2. Gan L, Liu Z, Cao W, Zhang Z, Sun C. FABP4 reversed the regulation of leptin on mitochondrial fatty acid oxidation in mice adipocytes. Sci Rep [Internet]. 2015 Aug 27 [cited 2021 Jun 14];5(1):13588. Available from: https://www.nature.com/articles/srep13588

3. Senga S, Kawaguchi K, Kobayashi N, Ando A, Fujii H. A novel fatty acid-binding protein 5-estrogenrelated receptor $\alpha$ signaling pathway promotes cell growth and energy metabolism in prostate cancer cells. Oncotarget [Internet]. 2018 Aug 3 [cited 2021 Jun 14];9(60):31753-70. Available from:

https://www.ncbi.nlm.nih.gov/pmc/articles/PMC6114981/

4. Ip W, Shao W, Song Z, Chen Z, Wheeler MB, Jin T. Liver-specific expression of dominant-negative transcription factor 7-like 2 causes progressive impairment in glucose homeostasis. Diabetes. 2015 Jun;64(6):1923-32.

5. Kim C-W, Addy C, Kusunoki J, Anderson NN, Deja S, Fu X, et al. Acetyl CoA Carboxylase Inhibition Reduces Hepatic Steatosis but Elevates Plasma Triglycerides in Mice and Humans: A Bedside to Bench Investigation. Cell Metabolism [Internet]. 2017 Aug 1 [cited 2021 Jun 14];26(2):394-406.e6. Available from: https://www.sciencedirect.com/science/article/pii/S1550413117304308

6. Hepatic De Novo Lipogenesis Is Present in Liver-Specific ACC1-Deficient Mice [Internet]. Molecular and Cellular Biology. [cited 2021 Jun 14]. Available from: https://journals.asm.org/doi/abs/10.1128/MCB.01122-06

7. Lee B, Fast AM, Zhu J, Cheng J-X, Buhman KK. Intestine-specific expression of acyl CoA:diacylglycerol acyltransferase 1 reverses resistance to diet-induced hepatic steatosis and obesity in Dgat1-/- mice. J Lipid Res [Internet]. 2010 Jul [cited 2021 Jun 14];51(7):1770-80. Available from: https://www.ncbi.nlm.nih.gov/pmc/articles/PMC2882751/

8. Reid BN, Ables GP, Otlivanchik OA, Schoiswohl G, Zechner R, Blaner WS, et al. Hepatic Overexpression of Hormone-sensitive Lipase and Adipose Triglyceride Lipase Promotes Fatty Acid Oxidation, Stimulates Direct Release of Free Fatty Acids, and Ameliorates Steatosis. J Biol Chem [Internet]. 2008 May 9 [cited 2021 Jun 14];283(19):13087-99. Available from: https://www.ncbi.nlm.nih.gov/pmc/articles/PMC2442319/

9. Ruiz-Villalba A, Mattiotti A, Gunst QD, Cano-Ballesteros S, van den Hoff MJB, Ruijter JM. Reference genes for gene expression studies in the mouse heart. Sci Rep [Internet]. 2017 Feb 2 [cited 2021 Jun 22];7(1):24. Available from: https://www.nature.com/articles/s41598-017-00043-9 